\title{
La poesia dell'altrove, nuove voci e percorsi italiani: la Compagnia delle poete
}

\section{Mia Lecomte}

\section{(2) OpenEdition}

\section{$\checkmark$ Journals}

\section{Edizione digitale}

URL: https://journals.openedition.org/cher/10923

DOI: 10.4000/cher.10923

ISSN: 2803-5992

\section{Editore}

Presses universitaires de Strasbourg

\section{Edizione cartacea}

Data di pubblicazione: 30 juin 2013

Paginazione: 55-61

ISBN: 978-2-35410-054-4

ISSN: 1968-035X

Notizia bibliografica digitale

Mia Lecomte, «La poesia dell'altrove, nuove voci e percorsi italiani: la Compagnia delle poete», reCHERches [Online], 10 | 2013, online dal 08 février 2022, consultato il 10 février 2022. URL: http:// journals.openedition.org/cher/10923 ; DOl: https://doi.org/10.4000/cher.10923

\section{(c) (i) (2)(2)}

Ce(tte) œuvre est mise à disposition selon les termes de la Licence Creative Commons Attribution Pas d'Utilisation Commerciale - Partage dans les Mêmes Conditions 4.0 International. 


\title{
La poesia dell'altrove, nuove voci e percorsi italiani: la Compagnia delle poete
}

\author{
Mia LeCOMTE \\ Scrittrice
}

\begin{abstract}
Tl 2010 è stato concordemente festeggiato come il ventennale della nascita della cosiddetta «letteratura della migrazione italofona», secondo una periodizzazione convenzionale che colloca nei primi anni Novanta gli albori di una nuova letteratura scritta da stranieri nella nostra lingua, non assimilabile a quella prodotta dall'élite di scrittori e letterati dei secoli passati, e giustificata invece dal flusso delle grandi migrazioni da cui l'Italia da allora è stata massicciamente interessata.

In questi vent'anni si è scritto e si è discusso molto intorno alla definizione di questa letteratura, alle sue caratteristiche e ragioni ultime, e ora si avverte la necessità profonda che il dibattito si evolva in una riflessione critica sulla sua qualità intrinseca, analizzata e considerata con studi approfonditi e calibrati sui singoli scrittori, che proprio per la varietà e l'eterogeneità di una produzione frutto di innesti e mescolanze linguistico-culturali, necessitano di ricognizioni individuali.

Diciamo che si è chiuso un periodo in cui tutti hanno fatto la loro parte: quegli autori che forse, senza la distinzione esotica della nuova etichetta, non avrebbero trovato facilmente uno spazio editoriale; gli editori, alcuni dei quali si sono ritagliati un settore di distinzione e dunque di sopravvivenza; gli accademici, che hanno sviscerato un argomento dalle molteplici implicazioni teoriche; e alcune frange politiche, che hanno cavalcato il fenomeno costituendo premi, concorsi, iniziative spesso pretestuose e disinformate,
\end{abstract}


che hanno intaccato a volte la credibilità qualitativa di queste nuove opere letterarie.

Ora sono soprattutto gli scrittori a lamentare i limiti di una categoria letteraria in cui si sentono arbitrariamente confinati. Quegli stessi scrittori, lo ripeto, che non hanno esitato a definirsi «migranti» - e parlo anche degli stanzialissimi meticci di seconda generazione, univocamente italofoni inseguendo in parte una speranza di mercato; e che ora hanno perlopiù realizzato i limiti di una visione angusta e provinciale - tutta italiana nella deliberata incomprensione dell'importanza epocale della letteratura di cui sono portatori, l'unica vera avanguardia a cui ci si possa al momento interessare - che impone diktat editoriali e commerciali ed etichette accademiche ormai logore, che finiscono per distorcere e umiliare la carica innovativa, eversiva delle loro modernissime voci.

Dopo vent'anni di analisi e dibattiti a senso unico, sembra essere giunto il momento di rovesciare la prospettiva di analisi, e di interrogarci sul ruolo, e addirittura sulla prospettiva di esistenza, delle letterature nazionali.

Conseguenza infatti dell'incessante trasmigrazione di anime plurilingui attraverso i flussi migratori, di un rimescolamento imprevedibile di identità polimorfe e frante, ovunque nel mondo si sta ingrossando l'arteria centrale delle letterature transnazionali e translingui, e si vanno impoverendo le vene stanche di quelle nazionali; sempre più chiaramente il nuovo tracciato artistico-letterario si sta disegnando come un corso impetuoso e travolgente in cui sfociano singoli affluenti ormai esangui, che avranno un destino attivo nella misura in cui sapranno far scorrere le proprie acque nei due sensi, regalando e attingendo forme e significati.

In attesa che tutto ciò venga riconosciuto e metabolizzato, gli scrittori migranti italofoni si ritrovano assemblati da anni in una sorta di zona franca, in attesa di una catalogazione definitiva, a discutere e a far discutere sull'identità e il destino della propria scrittura. Lontani dalla letteratura del paese d'origine, che non li riconosce come parte integrante della cultura nazionale, e ugualmente lontani per percorsi esistenziali e linguistici da quella del paese d'adozione, si trovano a condividere tutti promiscuamente un $\mathrm{CIE}^{1}$ delle parole in cui le aspettative e le proiezioni accademiche ed editoriali locali finiscono per comprometterne - con blandizie e abusi, sempre strumentalizzando la fragile riconoscibilità territoriale - la reale libertà di espressione.

1 Centro di Identificazione ed Espulsione. 
Per quel che riguarda la poesia, un motivato e consapevole interesse critico, anche per la congenita debolezza di genere - in questo caso infatti la marginalità è più completa, perché non entrano in gioco neanche le regole, sia pur distorte, del mercato editoriale della narrativa - è stato ancora più carente. Anzitutto si è creata una certa confusione nella periodizzazione della produzione poetica degli autori di cui ci si è venuti via via ad occupare. Se vogliamo prendere come cartina di tornasole l'inizio degli anni Novanta, vi troviamo infatti poeti che non condividono assolutamente lo stesso percorso storico e letterario, che non sono tutti ascrivibili alla specifica produzione dell'ultimo ventennio a cui ho accennato in principio. Innanzitutto ci sono autori arrivati in Italia trenta-quarant'anni prima, come l'ungherese Edith Bruch (le cui prime poesie in italiano risalgono ai primi anni Settanta), i brasiliani Murilo Mendes (che nel '68 pubblica la silloge in italiano Ipotesi, dopo undici anni di permanenza a Roma) e Marzia Theophilo (attiva in italiano dall'inizio degli anni Settanta), o Amelia Rosselli (che dopo il trasferimento a Roma, nel 1958 sceglie definitivamente l'italiano come lingua d'espressione poetica); poi quei poeti che avevano raggiunto almeno da un decennio qualità e riconoscibilità, come l'olandese Arnold de Vos (che nell'80 con la raccolta Poesie del deficit vince il Premio Piccolo Strega e Taormina), i brasiliani Heleno Oliveira (poeta in italiano a Firenze per tutti gli anni Ottanta) e Vera Lúcia de Oliveira (la prima raccolta, Geografie d'ombra, è pubblicata a Venezia nell'89), o l'iracheno Thea Laitef (morto a Roma nel '94, dopo aver pubblicato numerose poesie e un romanzo); i poeti che proprio all'inizio degli anni Novanta cominciano a raccogliere $\mathrm{i}$ primi onori, come l'albanese Gëzim Hajdari, che nel 1997 vince la sezione inediti del Premio Montale con la silloge Corpo presente; e infine i poeti che intraprendono proprio in quegli anni il cammino espressivo in italiano.

A tutt'oggi non esiste una raccolta antologica, o un saggio critico, che ordini cronologicamente in tal senso questa produzione poetica.

Ma più in generale - ad eccezione delle recenti pubblicazioni di Gianmario Lucini e Andrea Gazzoni rispettivamente su Arnold de Vos e Gëzim Hajdari - praticamente non esiste uno studio critico su un poeta della migrazione italofona o un'analisi testuale condotta con strumenti consoni a un approccio rigorosamente letterario ${ }^{2}$. L'unico interesse, lo ripeto, viene

2 Si veda tuttavia la Tesi di Laurea di Silvia Vajna de Pava, I miei occhi: sguardi incrociati. Gëzim Hajdari e la letteratura interculturale in lingua italiana, discussa nel 2005 presso l'Università di Milano (relatore Edoardo Esposito, correlatore Marco Modenesi) e vincintrice del Premio Eks\&Tra nel 2006. 
dagli studi di settore, e spesso all'interno di argomentazioni circoscritte, in cui la poesia risulta sempre la grande assente negli spazi di convegni accademici e seminari specialistici, dove raramente la lettura dei testi gode degli stessi diritti di ascolto dei corollari teorici.

Per quel che riguarda l'incontro e il confronto con la poesia italiana, inoltre, si può tranquillamente affermare che si tratta di esistenze che scorrono su binari paralleli, e le occasioni di conoscenza reciproca sono rare. Da parte della critica ufficiale c'è, in linea di massima, un'indifferenza pressoché totale nei confronti di questi nuovi poeti, non riconosciuti e compresi nella loro complessità culturale ed espressiva - anche perché lontani dall'omologazione tardo-ermetica e pseudo-avanguardistica che si continua ad imporre come imperante, e a tenere in vita a discapito di tutto il resto -, costantemente esclusi da antologie, classifiche, graduatorie, premi, festivals e incontri che non siano quelli della riserva a loro dedicata. E di conseguenza si comportano i poeti italiani, che spesso coincidono nel ruolo di critici, i quali non hanno alcun interesse ad interagire con questi nuovi autori in italiano per avviare un'arricchente, reciproca, collaborazione. C'è in generale un'insofferenza diffidente, dovuta in gran parte anche alla non-conoscenza, che porta a considerare questa poesia come «esotica»e «immatura». E soprattutto un'incapacità di soffermarsi sul futuro delle nostre letterature nazionali, come ho già scritto, e del loro retaggio identitario. Eppure sono riflessioni che hanno accompagnato da sempre la migranza poetica - basti solo pensare agli illustri referenti del ' 900 , primo fra tutti Josif Brodskij - e che si trovano lucidamente espressi già da Amelia Rosselli, che ho citato, di formazione trilingue, che ha subíto un destino di emigrazione, di andata e di ritorno, che ha profondamente segnato la sua vita e i suoi versi.

È proprio la Rosselli ad insistere sul provincialismo della società letteraria italiana rapportato invece alla variegata e poliglotta cultura degli scrittori che vi giungono da altrove, hanno letto altre letterature nella lingua originale, hanno saggiato e superato mode letterarie che investono il nostro paese con decennale ritardo; a ragionare sulla necessità di non lasciarsi condizionare dalla fonetica, rinnovando invece la lingua - a questo proposito nega i supposti «lapsus» attribuitile da Pasolini, che indica invece come invenzioni linguistiche conscie - che non predetermina lo stile né tantomeno l'ispirazione, e lavorando su quella «lingua da esiliata», che «anticipa il patchwork di un futuro italiano europeo». Ad affermare la necessità del «trauma» per creare un poeta originale, della ricerca della verità 
che deve guidare la creazione poetica, e ad avversare quel soggettivismo che riduce le tematiche a un incontro narcisistico con il privato.

Su questi aspetti, che costituiscono tutti la novità e la forza della cosiddetta attuale "poesia della migrazione» italofona, di cui la Rosselli è stata un'illustre precorritrice e una consapevole teorica, mi sono soffermata a lungo altrove; ma se li riprendo, in riferimento a una delle più grandi poetesse «italiane» del ' 900 , è per sottolineare che quella delle etichette e delle categorie in cui incasellare questi nuovi poeti italofoni è in realtà un falso problema, che fa perdere di vista il destino vitale e imprevedibile delle letterature de-nazionalizzate, che cerca di tenere in piedi a tutti i costi il canone perché faccia da baluardo contro l'apocalisse, in corso in tutti gli ambiti della nostra esistenza, di cui i poeti migranti sono i naturali e vitalissimi cantori.

Da queste premesse nella primavera del 2009 è nata la Compagnia delle poete. A comporla sono tutte straniere e italostraniere, almeno in parte italofone o residenti per un periodo dell'anno in Italia. Si tratta finora di una ventina di poete da diversi continenti, accomunate da una particolare storia personale di migranza e transnazionalità, affiancate nella realizzazione degli spettacoli da artiste e artisti - pittori, scultori, fotografi musicisti, ballerini e attori - che abbiano lavorato in ambito internazionale, muovendosi tra esperienze differenti.

L'idea è quella di una sorta di «orchestra» che armonizzi la poesia di ciascuna poeta, influenzata dalle diverse tradizioni linguistiche e culturali, in spettacoli in cui la parola è sostenuta e ampliata da molteplici linguaggi artistici. E secondo una struttura «modulare», che a seconda delle occasioni di esibizione e delle poete in scena, modifica e adatta di tappa in tappa la formula di base sulla quale è costruito lo spettacolo.

Per riportare la poesia al pubblico - scavalcate tutte le barriere critiche ed editoriali che la limitano e condizionano - restituendola alla sua originaria funzione di oralità condivisa.

La scelta di riunire solo poete straniere o italo-straniere non è una forma di ghettizzazione al contrario, ma è stata motivata dalla volontà di rappresentare una poesia che si discosta da molta della produzione italiana contemporanea, passata obbligatoriamente attraverso l'ermetismo e i fuochi tardivi di una prevedibile avanguardia, a cui a volte, per esigenze di ascolto e di mercato, si piegano gli stessi poeti migranti. Di dare voce a una poesia denazionalizzata, intrisa di altre lingue, che ha viaggiato e sa viaggiare, portatrice di storie e significati, più narrativa, mai soggettiva, spesso legata 
geograficamente e culturalmente all'oralità e, come nel caso di quella angloamericana, imparentata con il teatro. A cui manca anche la "memoria lunga» della tradizione poetica italiana, per cui la rinnova più spregiudicatamente, con una leggerezza provvida. E la ricaduta della destinazione performativa sulla creazione poetica, ha virato ancora di più i versi delle poete della Compagnia su tracciati di semplificata incisività.

La poesia femminile in questi anni sta dimostrando una sorprendente vivacità, sia quella migrante - e da Basili risulta che le poete e le narratrici hanno superato numericamente $i$ colleghi, un fenomeno in assoluta controtendenza rispetto al passato - ma anche quella «stanziale», e in particolare dialettale. Ė evidente che l'eccentricità al canone a lungo scontata dalle poete italiane ha sortito infine questa ricchezza, che combinata alla feconda marginalità della poesia migrante, ha permesso alle nuove poete in italiano i risultati raggiunti. La sovrapposizione di queste due eccentricità, cioé femminile e migrante, lontane dal nucleo di potere del canone, ha ritagliato uno spazio importante di innovazione dove la vera avanguardia vive di luce propria.

Ma l'idea di una Compagnia di sole donne non è partita solo da questo. Oltre che conseguente ad anni di amicizia e collaborazione tra le poete stesse, è nata anche per diffondere un'immagine della donna in generale diversa da quella svilita dai mass media, e più in particolare della donna con l'aggravante di straniera.

Il lavoro sui testi poetici destinato al palcoscenico ha poi rivelato una forte vicinanza carnale di questi versi al femminile, ha permesso di costruire spettacoli di una profonda coerenza testuale, attraversati da un filo rosso «violento» nella sua necessità, tenace. In scena una corporalità incarnata in molteplici forme, il legame stretto con la natura, la famiglia, l'impegno a tutto campo, un universo poetico desacralizzato dalla semplicità della presenza femminile abituata da sempre a gestire i propri molteplici ruoli, e così anche quello di poeta, senza enfasi.

E il multistrato polifonico che caratterizza la poesia migrante, di conseguenza, risulta rifranto ulteriormente nella pluralità dell'identità femminile.

Per quanto riguarda le «forme» della rappresentazione, dal momento che la «modularità» è insita nella formula sulla quale è stata costruita la Compagnia, sono attualmente in corso spettacoli differenti che coinvolgono questa o quella poeta con testi ogni volta diversi. 
Dopo il primo spettacolo-esperimento d'esordio, «Acromazie» - ovvero acrobazie leggere senza i contrasti e le differenze dei colori - nato inizialmente come video, $\mathrm{e}$ in cui le poete in scena recitavano anche i testi delle assenti in un canto condiviso che non specificava la maternità dei versi, è stata la volta di «Madrigne». Il titolo è un neologismo trasversale che vuole riunire in una sola immagine i ruoli diversi che si trova a ricoprire ogni donna - madre, madrina, matrigna... - per ricomporre il quadro di un universo femminile di imprevedibili matrioske.

La regia è un prodotto corale delle poete coinvolte, maturata sugli errori della prima esperienza, ed è costruita, con la supervisione di Vesna Stanić, intorno alla scenografia dell'artista tedesca Janine Von Thungen: scena vuota, quinte nere, le poete anch'esse in nero, luci essenziali, e un video utilizzato ad arte. Quest'ultimo è costituito dall'assemblaggio di immagini della quotidianità domestica di ognuna delle poete, a costruire delle microstorie che fanno da controcanto ai testi e alla musica dal vivo, per un disegno a tutto tondo dell'identità femminile. Come controtramatura sonora a riverbero delle immagini, c'è anche un mixaggio sonoro delle voci intrecciate di tutte le poete, e le registrazioni come voci fuori campo di quelle che non partecipano direttamente allo spettacolo.

La Compagnia sta attualmente lavorando a un'altra messinscena in sinergia con l'Accademia di Brera di Milano; lo spettacolo esordirà a marzo 2011 e seguirà, con gli altri, la propria storia di rappresentazioni. In un continuo work in progress per avvicinarsi sempre più - con le nuove poete che lavorano insieme, gli artisti diversi ogni volta coinvolti, il confronto con il pubblico durante esposizioni forse a volte anche premature e perfettibili - a quell'equilibrio esatto che permetta alla parola poetica di comunicare davvero ${ }^{3}$.

3 Per informazioni ulteriori riguardo all'iniziativa, le poete coinvolte, i testi, le foto, i video degli spettacoli, la rassegna stampa e gli ultimi appuntamenti, si rimanda al sito della Compagnia: http://www.compagniadellepoete.com/. 